OPEN ACCESS

Edited by:

Xiao-Lian Zhang,

Wuhan University, China

Reviewed by:

Werner Solbach,

University of Lübeck, Germany loana Diana Olaru,

University of London, United Kingdom

${ }^{*}$ Correspondence:

Lingqing $X_{U}$

lingqing_xu@126.com

Zhigang Xiong

xzghb@126.com

Ting Wang

981265056@qq.com

Xu Yuan

373082203@qq.com

Yong Gan

scswj2008@163.com

Ziyong Sun

zysun@tjh.tjmu.edu.cn

Feng Wang

fengwang@tjh.tjmu.edu.cn

Ying Luo

13349917282@163.com

Specialty section:

This article was submitted to

Microbial Immunology,

a section of the journal

Frontiers in Immunology

Received: 28 June 2021 Accepted: 07 October 2021 Published: 12 November 2021

Citation:

Luo Y, Xue Y, Lin Q,

Mao L, Tang G, Song H, Liu W, Wu S, Liu W, Zhou Y, Xu L, Xiong Z, Wang $T$, Yuan $X$, Gan $Y$, Sun $Z$ and Wang $F$ (2021) Diagnostic Model for Discrimination Between Tuberculous Meningitis and Bacterial Meningitis.

Front. Immunol. 12:731876. doi: 10.3389/fimmu.2021.731876

\section{Diagnostic Model for Discrimination Between Tuberculous Meningitis and Bacterial Meningitis}

\author{
Ying Luo ${ }^{1 *}$, Ying Xue ${ }^{2}$, Qun Lin ${ }^{1,3}$, Liyan Mao ${ }^{1}$, Guoxing Tang ${ }^{1}$, Huijuan Song ${ }^{1}$, Wei Liu ${ }^{1}$, \\ Shiji Wu ${ }^{1}$, Weiyong Liu ${ }^{1}$, Yu Zhou ${ }^{4}$, Lingqing $X u^{5 *}$, Zhigang Xiong ${ }^{1 *}$, Ting Wang ${ }^{1 *}$, \\ Xu Yuan ${ }^{1 *}$, Yong Gan $^{6 *}$, Ziyong Sun ${ }^{1 *}$ and Feng Wang ${ }^{1 *}$ \\ ${ }^{1}$ Department of Laboratory Medicine, Tongji Hospital, Tongji Medical College, Huazhong University of Science and \\ Technology, Wuhan, China, ${ }^{2}$ Department of Immunology, School of Basic Medicine, Tongji Medical College, Huazhong \\ University of Science and Technology, Wuhan, China, ${ }^{3}$ Laboratory Medicine Center, Nanfang Hospital, Southern Medical \\ University, Guangzhou, Guangdong, China, ${ }^{4}$ Department of Laboratory Medicine, Zhejiang Provincial People's Hospital, \\ People's Hospital of Hangzhou Medical College, Hangzhou, China, ${ }^{5}$ Qingyuan People's Hospital, The Sixth Affiliated Hospital \\ of Guangzhou Medical University, Qingyuan, China, ${ }^{6}$ Department of Social Medicine and Health Management, School of \\ Public Health, Tongji Medical College, Huazhong University of Science and Technology, Wuhan, China
}

Background: The differential diagnosis between tuberculous meningitis (TBM) and bacterial meningitis (BM) remains challenging in clinical practice. This study aimed to establish a diagnostic model that could accurately distinguish TBM from BM.

Methods: Patients with TBM or BM were recruited between January 2017 and January 2021 at Tongji Hospital (Qiaokou cohort) and Sino-French New City Hospital (Caidian cohort). The detection for indicators involved in cerebrospinal fluid (CSF) and T-SPOT assay were performed simultaneously. Multivariate logistic regression was used to create a diagnostic model.

Results: A total of 174 patients (76 TBM and 98 BM) and another 105 cases (39 TBM and 66 BM) were enrolled from Qiaokou cohort and Caidian cohort, respectively. Significantly higher level of CSF lymphocyte proportion while significantly lower levels of CSF chlorine, nucleated cell count, and neutrophil proportion were observed in TBM group when comparing with those in BM group. However, receiver operating characteristic (ROC) curve analysis showed that the areas under the ROC curve (AUCs) produced by these indicators were all under 0.8. Meanwhile, tuberculosis-specific antigen/ phytohemagglutinin (TBAg/PHA) ratio yielded an AUC of $0.889(95 \% \mathrm{Cl}, 0.840-0.938)$ in distinguishing TBM from BM, with a sensitivity of $68.42 \%(95 \% \mathrm{Cl}, 57.30 \%-77.77 \%)$ and a specificity of $92.86 \%(95 \% \mathrm{Cl}, 85.98 \%-96.50 \%)$ when a cutoff value of 0.163 was used. Consequently, we successfully established a diagnostic model based on the combination of TBAg/PHA ratio, CSF chlorine, CSF nucleated cell count, and CSF lymphocyte proportion for discrimination between TBM and BM. The established model showed good performance in differentiating TBM from BM (AUC: 0.949; 95\% Cl, $0.921-$ 0.978), with $81.58 \%(95 \% \mathrm{Cl}, 71.42 \%-88.70 \%)$ sensitivity and $91.84 \%(95 \% \mathrm{Cl}$, $84.71 \%-95.81 \%)$ specificity. The performance of the diagnostic model obtained in Qiaokou cohort was further validated in Caidian cohort. The diagnostic model in 
Caidian cohort produced an AUC of 0.923 (95\% Cl, 0.867-0.980) with 79.49\% (95\% Cl, 64.47\%-89.22\%) sensitivity and 90.91\% (95\% Cl, 81.55\%-95.77\%) specificity.

Conclusions: The diagnostic model established based on the combination of four indicators had excellent utility in the discrimination between TBM and BM.

Keywords: tuberculous meningitis, bacterial meningitis, differential diagnosis, TBAg/PHA ratio, diagnostic model

\section{INTRODUCTION}

Tuberculosis (TB), caused by Mycobacterium tuberculosis (MTB) infection, remains an ongoing and predominant health issue worldwide, with an estimated 10 million incident cases and 1.4 million deaths in 2019 globally (1). China is a high-TB-burden country with an average of 58 cases per 100,000 subjects (1). It was estimated that there were approximately 833,000 cases in 2019 (1). Tuberculous meningitis (TBM), the most severe type of extrapulmonary $\mathrm{TB}$, is prevalent in countries with high $\mathrm{TB}$ burden (2-4). Given that the manifestations of TBM can mimic other infectious diseases such as bacterial meningitis (BM), it is difficult for clinicians to diagnose TBM. Meanwhile, accurate and in-time diagnosis of TBM is critical for effective treatment. Consequently, there is an urgent need for developing accurate and rapid approaches for TBM diagnosis.

Unfortunately, the current diagnostic approaches for TBM have either low sensitivity (smear microscopy) or are timeconsuming (mycobacterial culture) (5). Detection of MTB DNA using molecular technologies such GeneXpert MTB/RIF and GeneXpert MTB/RIF Ultra provides quicker results than mycobacterial culture, yet more than half of cases cannot be bacteriologically confirmed (6). Specially, microscopy is rapid and widely available but could only detect $10 \%-20 \%$ cases (7-9). Mycobacterial culture and GeneXpert MTB/RIF have similar sensitivity of approximately $40 \%$ (6). High level of cerebrospinal fluid (CSF) adenosine deaminase was found in patients with TBM due to the activation of $\mathrm{T}$ lymphocytes in response to TB antigens (10). However, the sensitivity of the indicator is variable, and false-positive results were commonly noted in patients with HIV infection (10-12). Besides, T-SPOT.TB (T-SPOT), one of two commercially available interferon-gamma release assays, has been broadly used for diagnosis of MTB infection. Nevertheless, a recent meta-analysis conducted by Luo et al. (13) revealed a pooled sensitivity of $78 \%$ and specificity of $68 \%$ using peripheral blood T-SPOT and a pooled sensitivity of $76 \%$ and specificity of $88 \%$ using CSF T-SPOT in the diagnosis of TBM. Beyond that, indeterminate results are commonly noted, as sufficient sample volumes are usually unavailable, further limiting the use of CSF T-SPOT in clinical practice (14).

Given that microbiological confirmation is achieved in less than half of all cases due to the paucibacillary nature of the disease, the diagnosis of TBM has to rely on clinical, epidemiological, radiological, and routine laboratory features of patients. Consistent with this notion, the combination of different parameters might offer increased sensitivity and specificity over conventional approaches based on only one marker (15). As expected, some studies have tried to establish the diagnostic models targeting TBM diagnosis. For example, Yang et al. (16) found a four-parameter signature that could discriminate TBM from BM with a sensitivity of $98 \%$ and a specificity of $82 \%$. Another clinical prediction rule established by Vibha et al. (17) showed a sensitivity of $95.71 \%$ and a specificity of $97.63 \%$ in distinguishing TBM from BM. However, few models were validated in an independent cohort. Surprisingly, few studies incorporated T-SPOT assay into diagnostic models, while this assay has been shown to have some potential in the diagnosis of $\operatorname{TBM}(18,19)$. In addition, our previous studies have revealed that TB-specific antigen/phytohemagglutinin (TBAg/PHA) ratio of T-SPOT displayed improved performance on the diagnosis of extrapulmonary TB compared to directly using T-SPOT antigen spot numbers (20). This evidence suggested that the TBAg/PHA ratio may be an important laboratory marker that could be incorporated into TBM diagnostic model. In the present study, we attempted to establish a diagnostic model that had a prominent effect in discriminating TBM from BM.

\section{METHODS}

\section{Study Design}

The present study was conducted between January 2017 and January 2021. Adult patients over 17 years of age with TBM or BM were consecutively recruited from Tongji Hospital (Qiaokou cohort, the largest hospital in central China) and Sino-French New City Hospital (Caidian cohort, a branch hospital of Tongji Hospital). We evaluated the two datasets separately for training (Qiaokou cohort) and validation (Caidian cohort). TBM was diagnosed by positive MTB culture (Mycobacterial Growth Indicator Tube 960 and Lowenstein-Jensen media) and/or positive GeneXpert MTB/RIF (Cepheid, Sunnyvale, CA, USA) in CSF, with clinical symptoms and radiological characteristics suggestive of TBM (21-23). BM was diagnosed when pathogenic bacteria were isolated from the CSF using culture $(24,25)$. Matrixassisted laser desorption/ionization time-of-flight mass spectrometry (MALDI-TOF-MS) was used for bacteria identification. Since only patients with confirmed diagnosis were included in the current study, those who were unable to identify due to insufficient evidence or report missingness were excluded from the analysis. T-SPOT assay and other routine laboratory tests such as CSF cytology and biochemistry were performed simultaneously. Patients who received anti-TB treatment within 2 weeks prior to enrollment were excluded. The study was approved by the Ethics Committee of Tongji Hospital, Tongji Medical College, Huazhong University of Science and Technology. 


\section{T-SPOT Assay on Peripheral Blood}

Heparinized peripheral blood was collected and analyzed using T-SPOT assay (Oxford Immunotec, Oxford, UK) according to the manufacturer's instructions. Briefly, the isolated peripheral blood mononuclear cells (PBMCs) $\left(2.5 \times 10^{5}\right)$ were added to 96-well plates precoated with anti-interferon-gamma (anti-IFN$\gamma)$ antibody. Four wells were used for each subject: medium well, PHA well, early secreted antigenic target 6 (ESAT-6), and culture filtrate protein 10 (CFP-10) wells. Plates were incubated for $16-20 \mathrm{~h}$ at $37^{\circ} \mathrm{C}$ with $5 \% \mathrm{CO}_{2}$ and developed using an anti-IFN- $\gamma$ antibody conjugate and substrate to detect the presence of secreted IFN- $\gamma$. Spot-forming cells (SFCs) were counted with an automated ELISPOT reader (CTL Analyzers, Cleveland, $\mathrm{OH}$, USA). The test result was positive if ESAT-6 and/or CFP-10 minus negative control $\geq 6$ spots. The test result was negative if both ESAT- 6 minus negative control and CFP-10 minus negative control $\leq 5$ spots. Results were considered undetermined if the spot amounts in the PHA well were $<20$ or if spot amounts in the medium well were $>10$. We calculated the ratios of 1) ESAT-6 SFC to PHA SFC and 2) CFP-10 SFC to PHA SFC. The larger of the above two values was defined as the TBAg/PHA ratio of one participant (26).

\section{Statistical Analysis}

Continuous variables were expressed as means \pm standards deviation (SD) or median (interquartile range). Categorical variables were expressed as number (\%). Comparison was performed using Mann-Whitney $U$ test for continuous variables and chi-square test or Fisher's exact test for categorical variables. All statistical tests were two-sided.
Statistical significance was considered when $P<0.05$. In order to develop a diagnostic model, all variables with statistical significance were taken as candidates for multivariable logistic regression analyses, and the regression equation (diagnostic model) was obtained. The regression coefficients of the model were regarded as the weights for the respective variables, and a score for each patient was calculated. The performance of diagnostic models was evaluated by the receiver operating characteristic (ROC) curve analysis. Sensitivity, specificity, positive predictive value (PPV), negative predictive value (NPV), positive likelihood ratio (PLR), negative likelihood ratio (NLR), and accuracy, together with their 95\% confidence intervals (CI), were calculated. Data were analyzed using SPSS version 25.0 (SPSS, Inc., Chicago, IL, USA), GraphPad Prism version 8 (GraphPad Software, San Diego, CA, USA), and R 4.0.2 program (R Core Team).

\section{RESULTS}

\section{Clinical and Demographic Characteristics of the Included Participants}

A total of 174 patients, including 76 TBM and 98 BM, were enrolled in Qiaokou cohort. Another 105 subjects that consisted of 39 TBM and 66 BM were included in Caidian cohort. The main clinical and demographic data of the patients were summarized in Table 1. There was no statistical difference between TBM group and BM group in age and gender among these two cohorts. Headache and fever were the most common symptoms in the two groups. There was no statistical difference

TABLE 1 | Demographic and clinical characteristics of recruited participants.

\begin{tabular}{|c|c|c|c|c|c|c|c|}
\hline \multirow[t]{2}{*}{ Variables } & \multicolumn{2}{|c|}{ Qiaokou cohort (training set) } & \multirow[t]{2}{*}{$P^{*}$} & \multicolumn{2}{|c|}{ Caidian cohort (validation set) } & \multirow[t]{2}{*}{$P^{*}$} & \multirow[t]{2}{*}{$P^{\dagger}$} \\
\hline & TBM $(n=76)$ & $B M(n=98)$ & & TBM $(n=39)$ & BM $(n=66)$ & & \\
\hline Age, years & $44(27-58)$ & $42(30-54)$ & 0.926 & $45(29-59)$ & $39(26-54)$ & 0.294 & 0.689 \\
\hline Gender, male & $51(67.11 \%)$ & $69(70.41 \%)$ & 0.64 & $26(66.67 \%)$ & $43(65.15 \%)$ & 0.874 & 0.574 \\
\hline \multicolumn{8}{|l|}{ Symptoms } \\
\hline Headache & $62(81.58 \%)$ & $70(71.43 \%)$ & 0.121 & $30(76.92 \%)$ & $47(71.21 \%)$ & 0.523 & 0.637 \\
\hline Fever & $50(65.79 \%)$ & $53(54.08 \%)$ & 0.119 & $25(64.1 \%)$ & $37(56.06 \%)$ & 0.418 & 0.981 \\
\hline Vomiting & $26(34.21 \%)$ & $37(37.76 \%)$ & 0.629 & $16(41.03 \%)$ & $25(37.88 \%)$ & 0.749 & 0.634 \\
\hline Convulsion & $6(7.89 \%)$ & $9(9.18 \%)$ & 0.764 & $4(10.26 \%)$ & $5(7.58 \%)$ & 0.91 & 0.989 \\
\hline Irritability & $4(5.26 \%)$ & 8 (8.16\%) & 0.454 & $3(7.69 \%)$ & $4(6.06 \%)$ & 0.935 & 0.941 \\
\hline Weight loss & 7 (9.21\%) & $7(7.14 \%)$ & 0.619 & $3(7.69 \%)$ & 7 (10.61\%) & 0.883 & 0.670 \\
\hline \multicolumn{8}{|l|}{ Underlying condition or illness } \\
\hline Diabetes mellitus & 5 (6.58\%) & $10(10.2 \%)$ & 0.398 & $4(10.26 \%)$ & $6(9.09 \%)$ & 0.883 & 0.798 \\
\hline Hypertension & $3(3.95 \%)$ & $9(9.18 \%)$ & 0.176 & 5 (12.82\%) & 3 (4.55\%) & 0.245 & 0.821 \\
\hline Solid tumor & $9(11.84 \%)$ & $8(8.16 \%)$ & 0.418 & $4(10.26 \%)$ & $10(15.15 \%)$ & 0.476 & 0.359 \\
\hline Hematological malignancy & $6(7.89 \%)$ & $16(16.33 \%)$ & 0.097 & $3(7.69 \%)$ & $8(12.12 \%)$ & 0.699 & 0.587 \\
\hline Nephritis or kidney failure & $5(6.58 \%)$ & $5(5.1 \%)$ & 0.931 & $5(12.82 \%)$ & $4(6.06 \%)$ & 0.404 & 0.364 \\
\hline Virus hepatitis or cirrhosis & $10(13.16 \%)$ & $12(12.24 \%)$ & 0.857 & $6(15.38 \%)$ & $8(12.12 \%)$ & 0.635 & 0.868 \\
\hline Heart disease & $6(7.89 \%)$ & $8(8.16 \%)$ & 0.949 & $5(12.82 \%)$ & $6(9.09 \%)$ & 0.785 & 0.491 \\
\hline Organ transplantation & $2(2.63 \%)$ & $3(3.06 \%)$ & 0.772 & $1(2.56 \%)$ & $3(4.55 \%)$ & 0.988 & 0.937 \\
\hline HIV infection & $2(2.63 \%)$ & $0(0 \%)$ & 0.189 & $2(5.13 \%)$ & $1(1.52 \%)$ & 0.64 & 0.565 \\
\hline Immunosuppressive condition ${ }^{\ddagger}$ & $10(13.16 \%)$ & $15(15.31 \%)$ & 0.689 & $6(15.38 \%)$ & $12(18.18 \%)$ & 0.713 & 0.534 \\
\hline T-SPOT positivity & 65 (85.53\%) & $18(18.37 \%)$ & $<0.001$ & $31(79.49 \%)$ & $15(22.73 \%)$ & $<0.001$ & 0.528 \\
\hline
\end{tabular}

TBM, tuberculous meningitis; BM, bacterial meningitis. *Comparisons were performed between TBM group and BM group using Mann-Whitney U test, Chi-square test, or Fisher's exact test. ${ }^{\dagger}$ Comparisons were performed between Qiaokou and Caidian cohorts using Mann-Whitney $U$ test, Chi-square test, or Fisher's exact test. ${ }^{\ddagger}$ Patients who underwent chemotherapy or took immunosuppressants within 3 months. Data are presented as number (percentage) or medians (25th - 75th centiles). 
in underlying condition or illness between TBM and BM. The etiology information for BM was presented in Supplementary Table S1.

\section{Performance of Cerebrospinal Fluid Indicators for Differentiating Tuberculous Meningitis From Bacterial Meningitis}

The comparison between TBM group and BM group in CSF biochemical and cytological indicators was performed. It was observed that the levels of CSF chlorine, CSF nucleated cell count, and CSF neutrophil proportion were significantly lower in TBM group than those in BM group (Figure 1A). On the contrary, a significantly higher level of CSF lymphocyte proportion was noted in TBM group in comparison with that in BM group (Figure 1A). However, there was no statistical difference in the concentration of CSF glucose or total protein between TBM and BM patients (Figure 1A). ROC curve analysis was applied to evaluate the discriminatory performance of these indicators. It was found that CSF lymphocyte proportion produced an AUC of 0.732 (95\% CI, 0.656-0.808) while CSF neutrophil proportion yielded an AUC of 0.722 (95\% CI, 0.6450.799) in distinguishing TBM from BM (Table 2 and Figure 1B).

\section{Diagnostic Value of Tuberculosis-Specific Antigen/Phytohemagglutinin Ratio for Distinguishing Tuberculous Meningitis From Bacterial Meningitis}

We compared ESAT-6/PHA ratio, CFP-10/PHA ratio, and TBAg/PHA ratio between TBM patients and $\mathrm{BM}$ patients. It was found that ESAT-6/PHA ratio, CFP-10/PHA ratio, and TBAg/PHA ratio were significantly higher in TBM patients than those in BM patients (Figure 2A). ROC curve analysis demonstrated the AUC of ESAT-6/PHA ratio in discriminating TBM from BM to be 0.831 (95\% CI, 0.769-0.893). The cutoff value of 0.095 showed a sensitivity of $55.26 \%$ (95\% CI, $44.10 \%-$ $65.92 \%)$ and specificity of $91.84 \%$ (95\% CI, $84.71 \%-95.81 \%)$
A
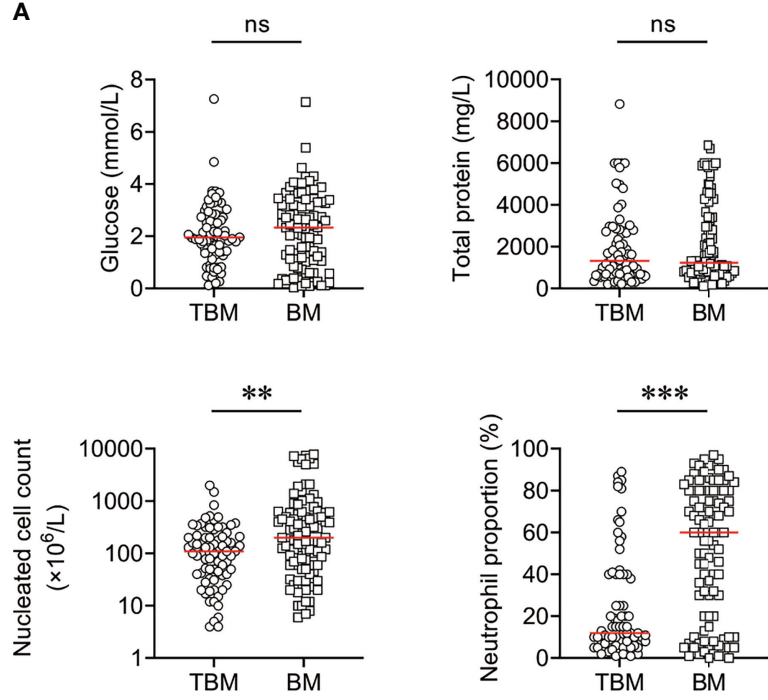
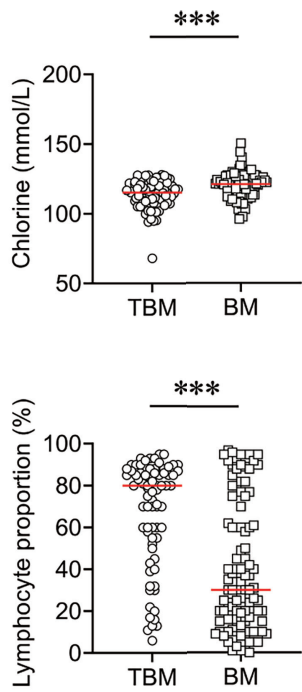

B

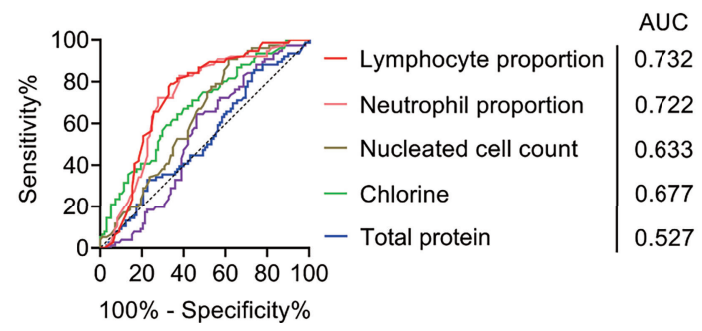

FIGURE 1 The performance of CSF biochemical and cytological indicators for distinguishing TBM from BM in Qiaokou cohort. (A) Scatter plots showing the levels of CSF indicators including glucose, total protein, chlorine, nucleated cell count, neutrophil proportion, and lymphocyte proportion in TBM patients ( $n=76$ ) and BM patients $\left(\mathrm{n}=98\right.$ ). Horizontal lines indicate the medians. ${ }^{* *} P<0.01 ;{ }^{* \star} P<0.001$; ns, no significance (Mann-Whitney $U$ test). (B) ROC curve analysis showing the performance of CSF indicators in distinguishing TBM from BM. TBM, tuberculous meningitis; BM, bacterial meningitis; ROC, receiver operating characteristic; CSF, cerebrospinal fluid; AUC, area under the ROC curve. 
TABLE 2 | The performance of CSF indicators for differentiating TBM from BM.

\begin{tabular}{|c|c|c|c|c|c|c|c|c|c|}
\hline Indicators & $\begin{array}{l}\text { Cutoff } \\
\text { value }\end{array}$ & $\begin{array}{c}\text { AUC } \\
(95 \% \mathrm{Cl})\end{array}$ & $\begin{array}{l}\text { Sensitivity } \\
\text { (95\% Cl) }\end{array}$ & $\begin{array}{l}\text { Specificity } \\
\text { (95\% Cl) }\end{array}$ & $\begin{array}{c}\text { PPV } \\
(95 \% \mathrm{Cl})\end{array}$ & $\begin{array}{c}\text { NPV } \\
(95 \% \mathrm{Cl})\end{array}$ & $\begin{array}{c}\text { PLR } \\
(95 \% \mathrm{CI})\end{array}$ & $\begin{array}{c}\text { NLR } \\
(95 \% \mathrm{Cl})\end{array}$ & Accuracy \\
\hline CSF chlorine (mmol/L) & 113 & $\begin{array}{c}0.677 \\
(0.598- \\
0.756)\end{array}$ & $\begin{array}{c}40.79 \% \\
(30.44 \%- \\
52.02 \%)\end{array}$ & $\begin{array}{c}80.61 \% \\
(71.69 \%- \\
87.22 \%)\end{array}$ & $\begin{array}{c}62.00 \% \\
(48.15 \%- \\
74.14 \%)\end{array}$ & $\begin{array}{c}63.71 \% \\
(54.95 \%- \\
71.64 \%)\end{array}$ & $\begin{array}{c}2.1 \\
(1.29- \\
3.42)\end{array}$ & $\begin{array}{l}0.73 \\
(0.6- \\
0.91)\end{array}$ & $63.22 \%$ \\
\hline $\begin{array}{l}\text { CSF nucleated cell } \\
\text { count }\left(\times 10^{6} / \mathrm{L}\right)\end{array}$ & 75 & $\begin{array}{c}0.633 \\
(0.551- \\
0.715)\end{array}$ & $\begin{array}{c}38.16 \% \\
(28.06 \%- \\
49.40 \%)\end{array}$ & $\begin{array}{c}69.39 \% \\
(59.68 \%- \\
77.64 \%)\end{array}$ & $\begin{array}{c}49.15 \% \\
(36.84 \%- \\
61.57 \%)\end{array}$ & $\begin{array}{c}59.13 \% \\
(49.99 \%- \\
67.68 \%)\end{array}$ & $\begin{array}{c}1.25 \\
(0.82- \\
1.88)\end{array}$ & $\begin{array}{c}0.89 \\
(0.72- \\
1.11)\end{array}$ & $55.75 \%$ \\
\hline $\begin{array}{l}\text { CSF neutrophil } \\
\text { proportion (\%) }\end{array}$ & 26 & $\begin{array}{c}0.722 \\
(0.645- \\
0.799)\end{array}$ & $\begin{array}{c}72.37 \% \\
(61.42 \%- \\
81.16 \%)\end{array}$ & $\begin{array}{c}72.45 \% \\
(62.88 \%- \\
80.32 \%)\end{array}$ & $\begin{array}{c}67.07 \% \\
(56.34 \%- \\
76.28 \%)\end{array}$ & $\begin{array}{c}77.17 \% \\
(67.61 \%- \\
84.56 \%)\end{array}$ & $\begin{array}{c}2.63 \\
(1.85- \\
3.73)\end{array}$ & $\begin{array}{c}0.38 \\
(0.26- \\
0.56)\end{array}$ & $72.41 \%$ \\
\hline $\begin{array}{l}\text { CSF Iymphocyte } \\
\text { proportion (\%) }\end{array}$ & 50 & $\begin{array}{c}0.732 \\
(0.656- \\
0.808)\end{array}$ & $\begin{array}{c}78.95 \% \\
(68.50 \%- \\
86.61 \%)\end{array}$ & $\begin{array}{c}66.33 \% \\
(56.51 \%- \\
74.91 \%)\end{array}$ & $\begin{array}{c}64.52 \% \\
(54.39 \%- \\
73.49 \%)\end{array}$ & $\begin{array}{c}80.25 \% \\
(70.30 \%- \\
87.46 \%)\end{array}$ & $\begin{array}{c}2.34 \\
(1.73- \\
3.17)\end{array}$ & $\begin{array}{c}0.32 \\
(0.2- \\
0.5)\end{array}$ & $71.84 \%$ \\
\hline
\end{tabular}

TBM, tuberculous meningitis; BM, bacterial meningitis; AUC, area under the curve; PPV, positive predictive value; NPV, negative predictive value; PLR, positive likelihood ratio; NLR, negative likelihood ratio; Cl, confidence interval; CSF, cerebrospinal fluid.
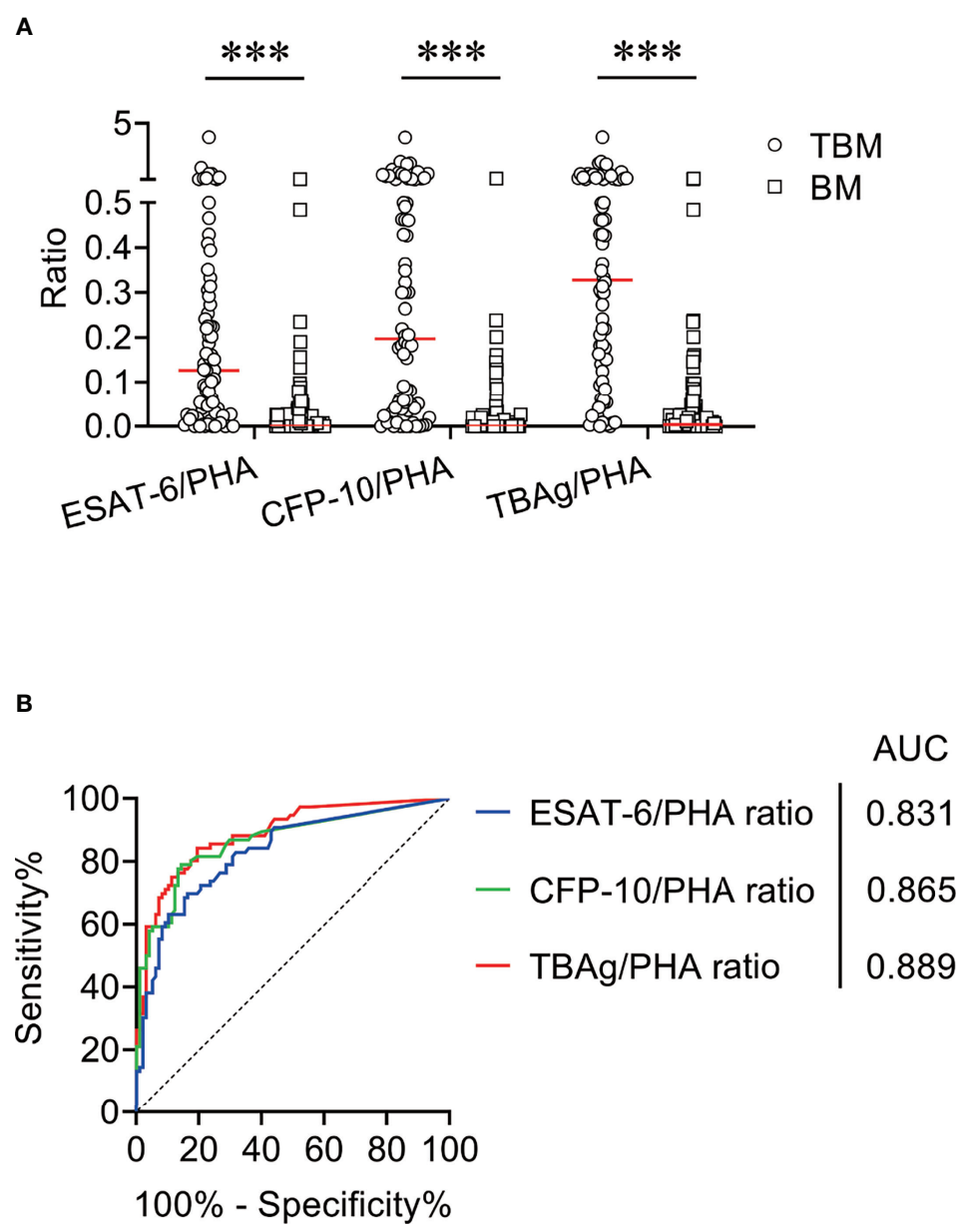

FIGURE 2 | The performance of TBAg/PHA ratio for distinguishing TBM from BM in Qiaokou cohort. (A) Scatter plots showing the levels of ESAT-6/PHA ratio, CFP10/PHA ratio, and TBAg/PHA ratio in TBM patients $(n=76)$ and BM patients $(n=98)$. Horizontal lines indicate the medians. ${ }^{* \star *} P<0.001$ (Mann-Whitney $U$ test). (B) ROC curve analysis showing the performance of ESAT-6/PHA ratio, CFP-10/PHA ratio, and TBAg/PHA ratio in distinguishing TBM from BM. TBM, tuberculous meningitis; BM, bacterial meningitis; ESAT-6, early secreted antigenic target 6; CFP-10, culture filtrate protein 10; PHA, phytohemagglutinin; TBAg, tuberculosisspecific antigen; ROC, receiver operating characteristic; AUC, area under the ROC curve. 
(Table 3 and Figure 2B). The AUC of CFP-10/PHA ratio in discriminating TBM from BM was 0.865 (95\% CI, 0.808-0.922). The sensitivity and specificity were 59.21\% (95\% CI, $47.98 \%-$ $69.56 \%$ ) and $90.82 \%$ (95\% CI, 83.46\%-95.09\%), respectively, with the threshold 0.11 (Table 3 and Figure 2B). Furthermore, the discriminatory power measured by the AUC for TBAg/PHA ratio was 0.889 (95\% CI, 0.840-0.938). When 0.163 was set as the cutoff value, the sensitivity and specificity were $68.42 \%$ (95\% CI, $57.30 \%-77.77 \%)$ and $92.86 \%$ (95\% CI, 85.98\%-96.50\%), respectively (Table 3 and Figure 2B).

\section{Establishing the Diagnostic Model in Discriminating Tuberculous Meningitis From Bacterial Meningitis}

We found that CSF biochemical and cytological indicators as well as TBAg/PHA ratio exhibited either limited or moderate value in discrimination between TBM and BM. In order to identify the possibility of combining CSF indicators and TBAg/PHA ratio to distinguish TBM from BM, we performed heatmap analysis and discovered the potential of combination of these indexes to differentiate TBM from BM (Figure 3). Next, we analyzed the cross set of indicators with significant differences in two groups. The overlap of seven indicators indicated the possible conjunct use for stratification (Figure 4). To establish the diagnostic model based on a combination of various indicators for distinguishing TBM from BM, all variables with statistical significance were used for multivariable logistic regression analysis. The diagnostic model was built as follows: $\mathrm{P}=1 /\left[1+\mathrm{e}^{-\left(11.512^{\star} \mathrm{TBAg} / \mathrm{PHA}\right.}\right.$ ratio $0.149^{*} \mathrm{CSF}$ chlorine $-0.001^{*} \mathrm{CSF}$ nucleated cell count $+0.041^{*} \mathrm{CSF}$ lymphocyte proportion +13.677$)] . P$, predictive value; e, natural logarithm. Venn diagram showed the overlap of these four parameters in TBM and $\mathrm{BM}$ groups and confirmed their appropriate combination
(Figure 5). The model presented an AUC of 0.949 (95\% CI, $0.921-0.978$ ) with a sensitivity of $81.58 \%$ (95\% CI, $71.42 \%-$ $88.70 \%)$ and specificity of $91.84 \%$ (95\% CI, 84.71\%-95.81\%) when 0.54 was used as the cutoff value (Table 4 and Figure 6).

\section{Validation of Established Diagnostic Model by an Independent Cohort}

Another independent set (Caidian cohort) was included to validate our established model. Similar to the training cohort, the diagnostic model could effectively discriminate TBM from $\mathrm{BM}$ in the validation cohort. According to the cutoff value obtained in the training cohort, the diagnostic model showed an accuracy of $86.67 \%$, with a sensitivity of $79.49 \%$ (95\% CI, $64.47 \%-89.22 \%)$ and a specificity of $90.91 \%$ (95\% CI, $81.55 \%-$ $95.77 \%$ ) in discriminating $\mathrm{TBM}$ from BM (Table 4 and Figure 7).

\section{DISCUSSION}

Discrimination between TBM and BM remains a challenge in clinical practice. The current bacteriological tests had low sensitivity for the diagnosis of TBM $(27,28)$. Besides, the lack of accessible and timely methods also contributed to a delay in diagnosis and subsequent morbidity and mortality for TBM patients, particularly those in resource-limited settings (29). Although the potential biomarkers of proteome (30), metabolome (31), and transcriptome (32) have been identified for TBM diagnosis in recent years, these markers have not been sufficiently verified. In this study, we compared the laboratory features including CSF indexes and TBAg/PHA ratio in T-SPOT assay among patients with these two types of meningitis.

TABLE 3 | The performance of TBAg/PHA ratio for distinguishing between TBM and BM.

\begin{tabular}{|c|c|c|c|c|c|c|c|c|c|}
\hline Indicators & $\begin{array}{l}\text { Cutoff } \\
\text { value }\end{array}$ & $\begin{array}{c}\text { AUC } \\
(95 \% \mathrm{Cl})\end{array}$ & $\begin{array}{l}\text { Sensitivity } \\
\text { (95\% CI) }\end{array}$ & $\begin{array}{l}\text { Specificity } \\
\text { (95\% CI) }\end{array}$ & $\begin{array}{c}\text { PPV } \\
(95 \% \mathrm{Cl})\end{array}$ & $\begin{array}{c}\text { NPV } \\
(95 \% \mathrm{Cl})\end{array}$ & $\begin{array}{c}\text { PLR } \\
(95 \% \mathrm{Cl})\end{array}$ & $\begin{array}{c}\text { NLR } \\
(95 \% \mathrm{Cl})\end{array}$ & Accuracy \\
\hline $\begin{array}{l}\text { ESAT-6/PHA } \\
\text { ratio }\end{array}$ & 0.095 & $\begin{array}{c}0.831(0.769- \\
0.893)\end{array}$ & $\begin{array}{c}55.26 \%(44.10 \%- \\
65.92 \%)\end{array}$ & $\begin{array}{c}91.84 \%(84.71 \%- \\
95.81 \%)\end{array}$ & $\begin{array}{c}84.00 \%(71.49 \%- \\
91.66 \%)\end{array}$ & $\begin{array}{c}72.58 \%(64.14 \%- \\
79.67 \%)\end{array}$ & $\begin{array}{c}6.77(3.38- \\
13.55)\end{array}$ & $\begin{array}{c}0.49(0.38- \\
0.63)\end{array}$ & $75.86 \%$ \\
\hline $\begin{array}{l}\text { CFP-10/PHA } \\
\text { ratio }\end{array}$ & 0.11 & $\begin{array}{c}0.865(0.808- \\
0.922)\end{array}$ & $\begin{array}{c}59.21 \%(47.98 \%- \\
69.56 \%)\end{array}$ & $\begin{array}{c}90.82 \%(83.46 \%- \\
95.09 \%)\end{array}$ & $\begin{array}{c}83.33 \%(71.26 \%- \\
90.98 \%)\end{array}$ & $\begin{array}{c}74.17 \%(65.67 \%- \\
81.16 \%)\end{array}$ & $\begin{array}{c}6.45(3.37- \\
12.35)\end{array}$ & $\begin{array}{c}0.45(0.34- \\
0.59)\end{array}$ & $77.01 \%$ \\
\hline $\begin{array}{l}\text { TBAg/PHA } \\
\text { ratio }\end{array}$ & 0.163 & $\begin{array}{c}0.889(0.840- \\
0.938)\end{array}$ & $\begin{array}{c}68.42 \%(57.30 \%- \\
77.77 \%)\end{array}$ & $\begin{array}{c}92.86 \%(85.98 \%- \\
96.50 \%)\end{array}$ & $\begin{array}{c}88.14 \%(77.48 \%- \\
94.13 \%)\end{array}$ & $\begin{array}{c}79.13 \%(70.82 \%- \\
85.56 \%)\end{array}$ & $\begin{array}{c}9.58(4.62- \\
19.88)\end{array}$ & $\begin{array}{c}0.34(0.24- \\
0.48)\end{array}$ & $82.18 \%$ \\
\hline
\end{tabular}

TBAg, tuberculosis antigen; PHA, phytohemagglutinin; TBM, tuberculous meningitis; BM, bacterial meningitis; AUC, area under the Curve; PPV, positive predictive value; NPV, negative predictive value; PLR, positive likelihood ratio; NLR, negative likelihood ratio; CI, confidence interval; ESAT-6, early secreted antigenic target 6; CFP-10, culture filtrate protein 10.

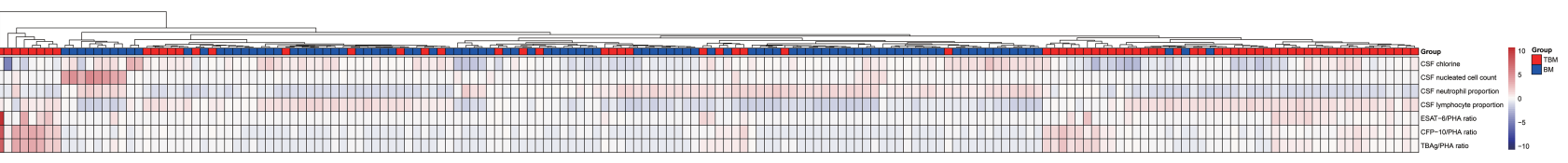

FIGURE 3 | The cluster analysis of various indicators in TBM group and BM group. Heatmap showing the cluster analysis of CSF chlorine, CSF nucleated cell count, CSF neutrophil proportion, CSF lymphocyte proportion, ESAT-6/PHA ratio, CFP-10/PHA ratio, and TBAg/PHA ratio in TBM patients ( $\mathrm{n}=76$ ) and BM patients ( $n=98$ ). Each rectangle indicates a result of a patient. TBM, tuberculous meningitis; BM, bacterial meningitis; CSF, cerebrospinal fluid; ESAT-6, early secreted antigenic target 6; CFP-10, culture filtrate protein 10; PHA, phytohemagglutinin; TBAg, tuberculosis-specific antigen. 


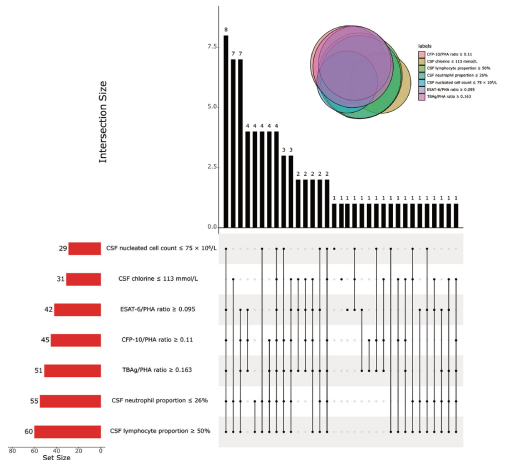

B

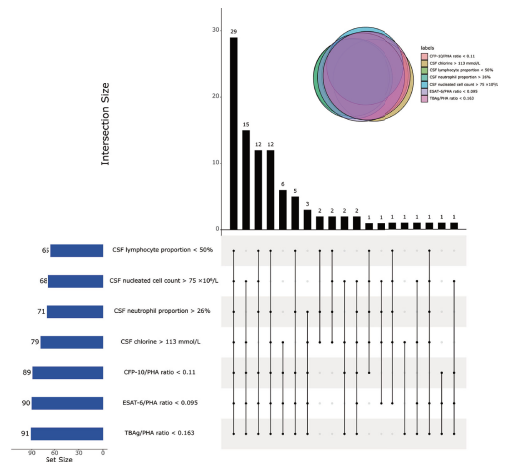

FIGURE 4 | The cross set between various indicators in TBM group and BM group. (A) Upset plot showing the cross set between various indicators in TBM group. (B) Upset plot showing the cross set between various indicators in BM group. TBM, tuberculous meningitis; BM, bacterial meningitis; CSF, cerebrospinal fluid; ESAT-6, early secreted antigenic target 6; CFP-10, culture filtrate protein 10; PHA, phytohemagglutinin; TBAg, tuberculosis-specific antigen.

Importantly, we successfully established and verified a diagnostic model with good performance for distinguishing TBM from BM.

One of the most important reasons why TBM diagnosis is difficult is that the positive rate of microbiological detection methods was low in CSF. It is generally viewed that the sensitivity of acid-fast stain, MTB culture, and GeneXpert MTB/RIF in patients with TBM was much lower than that in patients with pulmonary TB (33-36). It is because that the concentration of MTB pathogen is low in CSF when TBM occurs. Thus, the diagnosis of TBM based on the combination of routine laboratory features is necessary at present. Sometimes, clinicians had to prescribe diagnostic anti-TB therapy to help TBM diagnosis, and missed diagnosis and misdiagnosis are inevitable in real clinical practice. The present study built a four-indicator diagnostic model based on combining CSF indexes and TBAg/PHA ratio. Notably, this four-indicator model had a prominent effect on differentiating TBM from $\mathrm{BM}$, yielding an accuracy of more than $85 \%$, with the AUC more than 0.9. Hereafter, the performance of the model was successfully verified in an independent cohort, further supporting the evidence that the model may have wide applicability in TBM diagnosis in real clinical practice.

It is noteworthy that rare studies had included T-SPOT assay to build a diagnostic model previously. We speculated the reason for this may be that the operating procedures of T-SPOT assay are complicated, and it is generally viewed that the repeatability and reproducibility of the method need to be further verified. However, we have spent a lot of effort in the standardization of T-SPOT assay. We have first emphasized that the substrate incubation time, peripheral blood mononuclear cell counting, and the setting of ELISPOT reader parameters (such as exposure time and sensitivity) are the key factors affecting SFC results of antigen and PHA in T-SPOT assay $(20,37)$. In addition, we first put forward the concept of TBAg/PHA ratio and found that the performance of TBAg/PHA ratio is better than directly using $\mathrm{T}$-SPOT antigen results not only in distinguishing active tuberculosis (ATB) from latent tuberculosis infection (LTBI) but also in differential diagnosis of pulmonary and extrapulmonary TB $(20,26,37-41)$. We also first put forward that $1 \times 10^{5}$ was the optimal number of pleural fluid mononuclear cells for performing pleural fluid T-SPOT and that the mean spot size of ESAT- 6 has an adjunctive role in the diagnosis of ATB $(42,43)$. Thus, our previously published findings suggest that we have accumulated excellent experience in performing T-SPOT assay. To the best of our knowledge, this is the first study to establish the diagnostic model based on the combination of TBAg/PHA ratio and other indicators to discriminate TBM from BM. In view of the fact that TBAg/ PHA ratio is a further calculation of T-SPOT results, the model does not increase additional costs.

Several limitations should be noticed in our study. First, the sample size in each cohort, especially the validation cohort, was not large enough. Additional studies including larger cases are required to determine the performance of the established model. Second, the patient population in the current study may not be representative of patients with meningitis in general. In clinical practice, meningitis can have a wider range of causes including viral and fungal infection, as well as autoimmune diseases. Patients with these conditions were not included in this study. Therefore, further investigation with the inclusion of these populations is needed to determine the real utility of the established model. Third, to ensure the reliability of inclusion, our study only included patients with microbiologically diagnosed TBM. However, the diagnosis for clinically identified TBM (probable or possible TBM) was more crucial $(6,44)$. The validation of the established model in diagnosing probable or possible TBM is also warranted in the future. Fourth, it is important to note that the cost-effectiveness of the application of the models in real-world clinical settings should be evaluated in the future. Finally, it is undeniable that the combination of various measurements not only increases additional manpower, material resources, and costs but also increases the complications and difficulties in the implementation. However, the reality at present is that new technologies have not been fully proven effective and 
A

CSF nucleated cell count $\leq 75 \times 10^{6} / \mathrm{L} \quad$ CSF lymphocyte proportion $\geq 50 \%$

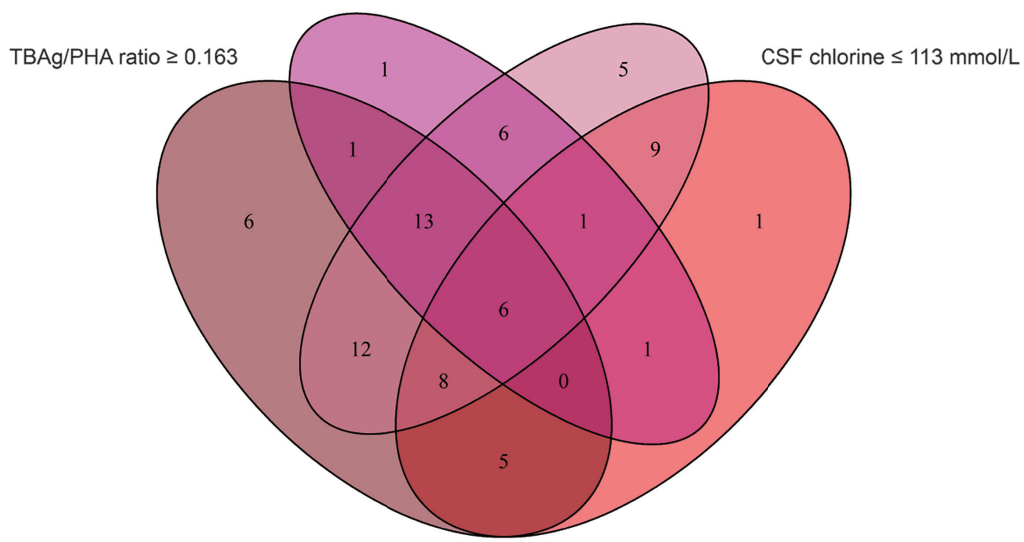

B

CSF nucleated cell count $>75 \times 10^{6} / \mathrm{L} \quad$ CSF lymphocyte proportion $<50 \%$

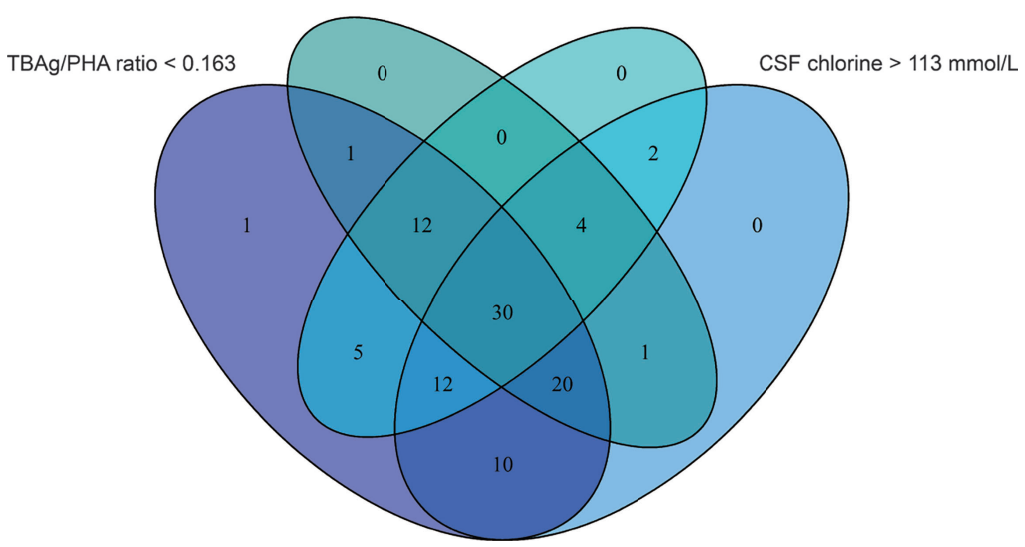

FIGURE 5 | The cross set between various indicators in TBM group and BM group. (A) Venn diagrams showing the overlap of TBAg/PHA ratio, CSF nucleated cell count, CSF lymphocyte proportion, and CSF chlorine in TBM patients. (B) Venn diagrams showing the overlap of TBAg/PHA ratio, CSF nucleated cell count, CSF lymphocyte proportion, and CSF chlorine in BM patients. TBM, tuberculous meningitis; BM, bacterial meningitis; TBAg, tuberculosis-specific antigen; PHA, phytohemagglutinin; CSF, cerebrospinal fluid.

TABLE 4 | The performance of diagnostic model for discriminating TBM from BM.

\begin{tabular}{lcr}
\hline Parameters & Training set & Validation set \\
\hline Cutoff value & 0.54 & 0.54 \\
AUC $(95 \% \mathrm{Cl})$ & $0.949(0.921-0.978)$ & $0.923(0.867-0.980)$ \\
Sensitivity $(95 \% \mathrm{Cl})$ & $81.58 \%(71.42 \%-88.70 \%)$ & $79.49 \%(64.47 \%-89.22 \%)$ \\
Specificity $(95 \% \mathrm{Cl})$ & $91.84 \%(84.71 \%-95.81 \%)$ & $90.91 \%(81.55 \%-95.77 \%)$ \\
PPV $(95 \% \mathrm{Cl})$ & $88.57 \%(79.04 \%-94.09 \%)$ & $83.78 \%(68.86 \%-92.35 \%)$ \\
NPV $(95 \% \mathrm{Cl})$ & $86.54 \%(78.67 \%-91.81 \%)$ & $88.24 \%(78.47 \%-93.92 \%)$ \\
PLR $(95 \% \mathrm{Cl})$ & $9.99(5.1-19.58)$ & $8.74(4.01-19.06)$ \\
NLR $(95 \% \mathrm{Cl})$ & $0.2(0.12-0.32)$ & $0.23(0.12-0.42)$ \\
Accuracy & $87.36 \%$ & $86.67 \%$
\end{tabular}

TBM, tuberculous meningitis; BM, bacterial meningitis; AUC, area under the curve; PPV, positive predictive value; NPV, negative predictive value; PLR, positive likelihood ratio; NLR, negative likelihood ratio; $\mathrm{Cl}$, confidence interval. 
A

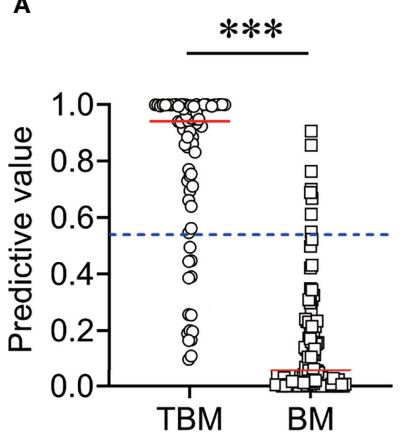

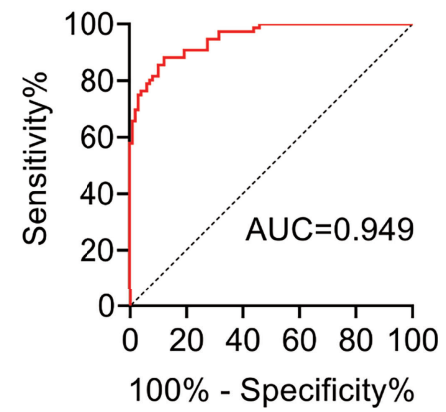

FIGURE 6 | The performance of diagnostic model for discriminating TBM from BM in Qiaokou cohort. (A) Scatter plots showing the predictive value of the diagnostic model in TBM patients $(n=76)$ and BM patients $(n=98)$. Horizontal lines indicate the medians. ${ }^{\star \star \star} P<0.001$ (Mann-Whitney $U$ test). Blue dotted lines indicate the cutoff value in distinguishing these two groups. (B) ROC curve analysis showing the performance of the diagnostic model in distinguishing TBM from BM. ROC, receiver operating characteristic; AUC, area under the ROC curve.

A

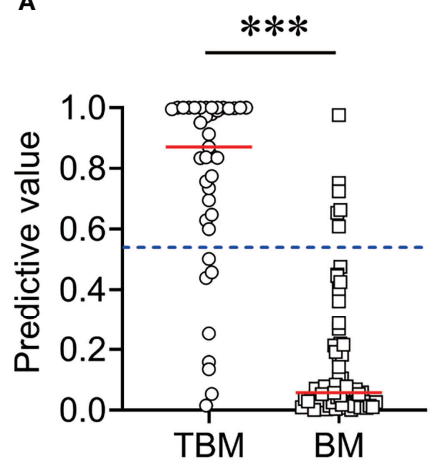

B

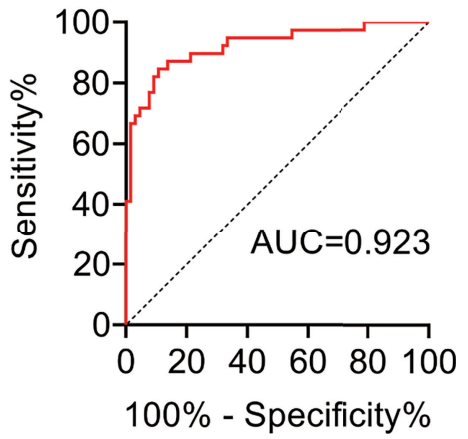

FIGURE 7 | The performance of diagnostic model for discriminating TBM from BM in Caidian cohort. (A) Scatter plots showing the predictive value of the diagnostic model in TBM patients $(n=39)$ and BM patients $(n=66)$. Horizontal lines indicate the medians. ${ }^{\star \star \star} P<0.001$ (Mann-Whitney $U$ test). Blue dotted lines indicate the cutoff value in distinguishing these two groups. (B) ROC curve analysis showing the performance of the diagnostic model in distinguishing TBM from BM. ROC, receiver operating characteristic; $\mathrm{AUC}$, area under the ROC curve.

traditional technologies cannot meet clinical needs; the obstacles brought by the joint applications are worth to overcome.

Collectively, our study uncovered and established a novel diagnostic model based on the combination of four indicators with excellent utility in distinguishing TBM from BM. Moreover, our model would have potential in facilitating timely initiation of anti-tuberculosis treatment and improving patients' outcome.

\section{DATA AVAILABILITY STATEMENT}

The original contributions presented in the study are included in the article/Supplementary Material. Further inquiries can be directed to the corresponding authors.

\section{ETHICS STATEMENT}

The studies involving human participants were reviewed and approved by the Ethics Committee of Tongji Hospital, Tongji Medical College, Huazhong University of Science and Technology. The patients/participants provided their written informed consent to participate in this study.

\section{AUTHOR CONTRIBUTIONS}

YL had the original idea of the study. YL conceived and designed the research. YL interpreted data and wrote the article. YL, YX, QL, LM, GT, HS, and WL contributed to the acquisition of 
clinical data. YL, SW, WYL, YZ, LX, ZX, TW, and XY recruited the participants, performed experiments, and analyzed data. YL, YG, FW, and ZS contributed to the revision of the article. All authors contributed to the article and approved the submitted version.

\section{FUNDING}

This work was supported by grants from the Fundamental Research Funds for the Central Universities (no. 2021yjsCXCY088), the Special Foundation for National Science and Technology Basic Research Program of China (no. 2019FY101206), the National Natural Science Foundation (no. 81902132), and the Qingyuan

\section{REFERENCES}

1. World Health Organization. Global Tuberculosis Report 2020. Geneva, Switzerland (2020). World Health Organization. Available at: https:// appswhoint/iris/rest/bitstreams/1312164/retrieve. 14 October 2020.

2. Pang Y, An J, Shu W, Huo F, Chu N, Gao M, et al. Epidemiology of Extrapulmonary Tuberculosis Among Inpatients, China, 2008-2017. Emerg Infect Dis (2019) 25(3):457-64. doi: 10.3201/eid2503.180572

3. Seddon JA, Tugume L, Solomons R, Prasad K, Bahr NCTuberculous Meningitis International Research C. The Current Global Situation for Tuberculous Meningitis: Epidemiology, Diagnostics, Treatment and Outcomes. Wellcome Open Res (2019) 4:167. doi: 10.12688/wellcomeopenres.15535.1

4. Robertson FC, Lepard JR, Mekary RA, Davis MC, Yunusa I, Gormley WB, et al. Epidemiology of Central Nervous System Infectious Diseases: A Meta-Analysis and Systematic Review With Implications for Neurosurgeons Worldwide. J Neurosurg (2018) 1:1-20. doi: 10.3171/ 2017.10.JNS17359

5. Heemskerk AD, Donovan J, Thu DDA, Marais S, Chaidir L, Dung VTM, et al. Improving the Microbiological Diagnosis of Tuberculous Meningitis: A Prospective, International, Multicentre Comparison of Conventional and Modified Ziehl-Neelsen Stain, Genexpert, and Culture of Cerebrospinal Fluid. J Infect (2018) 77(6):509-15. doi: 10.1016/j.jinf.2018.09.003

6. Donovan J, Thu DDA, Phu NH, Dung VTM, Quang TP, Nghia HDT, et al. Xpert MTB/RIF Ultra Versus Xpert MTB/RIF for the Diagnosis of Tuberculous Meningitis: A Prospective, Randomised, Diagnostic Accuracy Study. Lancet Infect Dis (2020) 20(3):299-307. doi: 10.1016/S1473-3099(19)30649-8

7. Bahr NC, Boulware DR. Methods of Rapid Diagnosis for the Etiology of Meningitis in Adults. biomark Med (2014) 8(9):1085-103. doi: 10.2217/ bmm.14.67

8. Bahr NC, Tugume L, Rajasingham R, Kiggundu R, Williams DA, Morawski B, et al. Improved Diagnostic Sensitivity for Tuberculous Meningitis With Xpert ((R)) MTB/RIF of Centrifuged CSF. Int J Tuberc Lung Dis (2015) 19 (10):1209-15. doi: 10.5588/ijtld.15.0253

9. Bahr NC, Marais S, Caws M, van Crevel R, Wilkinson RJ, Tyagi JS, et al. Genexpert MTB/Rif to Diagnose Tuberculous Meningitis: Perhaps the First Test But Not the Last. Clin Infect Dis (2016) 62(9):1133-5. doi: 10.1093/cid/ciw083

10. Pormohammad A, Riahi SM, Nasiri MJ, Fallah F, Aghazadeh M, Doustdar F and Pouriran R. Diagnostic Test Accuracy of Adenosine Deaminase for Tuberculous Meningitis: A Systematic Review and Meta-Analysis. J Infect (2017) 74(6):545-54. doi: 10.1016/j.jinf.2017.02.012

11. Corral I, Quereda C, Navas E, Martin-Davila P, Perez-Elias MJ, Casado JL, et al. Adenosine Deaminase Activity in Cerebrospinal Fluid of HIV-Infected Patients: Limited Value for Diagnosis of Tuberculous Meningitis. Eur J Clin Microbiol Infect Dis (2004) 23(6):471-6. doi: 10.1007/s10096-004-1110-z

12. Ekermans P, Duse A and George J. The Dubious Value of Cerebrospinal Fluid Adenosine Deaminase Measurement for the Diagnosis of Tuberculous Meningitis. BMC Infect Dis (2017) 17(1):104. doi: 10.1186/s12879-0172221-3
People's Hospital Medical Scientific Research Fund Project (no. 20190209).

\section{ACKNOWLEDGMENTS}

The authors thank the clinical immunology and microbiology laboratories of Tongji Hospital for technical assistance as well as the patients and their families.

\section{SUPPLEMENTARY MATERIAL}

The Supplementary Material for this article can be found online at: https://www.frontiersin.org/articles/10.3389/fimmu.2021. 731876/full\#supplementary-material

13. Luo Y, Xue Y, Guo X, Lin Q, Mao L, Tang G, et al. Diagnostic Accuracy of TSPOT.TB Assay for Tuberculous Meningitis: An Updated Meta-Analysis. Front Neurol (2020) 11:866. doi: 10.3389/fneur.2020.00866

14. Kim SH, Cho OH, Park SJ, Lee EM, Kim MN, Lee SO, et al. Rapid Diagnosis of Tuberculous Meningitis by T Cell-Based Assays on Peripheral Blood and Cerebrospinal Fluid Mononuclear Cells. Clin Infect Dis (2010) 50(10):134958. doi: 10.1086/652142

15. Youssef FG, Afifi SA, Azab AM, Wasfy MM, Abdel-Aziz KM, Parker TM, et al. Differentiation of Tuberculous Meningitis From Acute Bacterial Meningitis Using Simple Clinical and Laboratory Parameters. Diagn Microbiol Infect Dis (2006) 55 (4):275-8. doi: 10.1016/j.diagmicrobio.2006.01.027

16. Yang Y, Qu XH, Zhang KN, Wu XM, Wang XR, Wen A, et al. A Diagnostic Formula for Discrimination of Tuberculous and Bacterial Meningitis Using Clinical and Laboratory Features. Front Cell Infect Microbiol (2019) 9:448. doi: 10.3389/fcimb. 2019.00448

17. Vibha D, Bhatia R, Prasad K, Srivastava MV, Tripathi M, Kumar G and Singh MB. Validation of Diagnostic Algorithm to Differentiate Between Tuberculous Meningitis and Acute Bacterial Meningitis. Clin Neurol Neurosurg (2012) 114(6):639-44. doi: 10.1016/j.clineuro.2011.12.033

18. Park KH, Lee MS, Kim SM, Park SJ, Chong YP, Lee SO, et al. Diagnostic Usefulness of T-Cell Based Assays for Tuberculous Meningitis in HIVUninfected Patients. J Infect (2016) 72(4):486-97. doi: 10.1016/j.jinf.2016. 01.012

19. Park KH, Cho OH, Lee EM, Lee SO, Choi SH, Kim YS, et al. T-Cell-Based Assays on Cerebrospinal Fluid and Pbmcs for Rapid Diagnosis of TB Meningitis in Non-HIV Patients. Eur Respir J (2012) 39(3):768-70. doi: 10.1183/09031936.00098111

20. Wang F, Yu J, Zhou Y, Luo Y, Wu S, Huang M, et al. The Use of TB-Specific Antigen/Phytohemagglutinin Ratio for Diagnosis and Treatment Monitoring of Extrapulmonary Tuberculosis. Front Immunol (2018) 9:1047. doi: 10.3389/ fimmu.2018.01047

21. World Health Organization. Implementing Tuberculosis Diagnostics: A Policy Framework. Geneva, Switzerland: World Health Organization (2015). Available at: https://appswhoint/iris/rest/bitstreams/720125/retrieve. 24 April 2015.

22. Arshad A, Dayal S, Gadhe R, Mawley A, Shin K, Tellez D, et al. Analysis of Tuberculosis Meningitis Pathogenesis, Diagnosis, and Treatment. J Clin Med (2020) 9(9):2962. doi: $10.3390 / \mathrm{jcm} 9092962$

23. Bourgi K, Fiske C and Sterling TR. Tuberculosis Meningitis. Curr Infect Dis Rep (2017) 19(11):39. doi: 10.1007/s11908-017-0595-4

24. Brouwer MC, Thwaites GE, Tunkel AR, van de Beek D. Dilemmas in the Diagnosis of Acute Community-Acquired Bacterial Meningitis. Lancet (2012) 380(9854):1684-92. doi: 10.1016/S0140-6736(12)61185-4

25. van de Beek D, Cabellos C, Dzupova O, Esposito S, Klein M, Kloek AT, et al. ESCMID Guideline: Diagnosis and Treatment of Acute Bacterial Meningitis. Clin Microbiol Infect (2016) 22 Suppl 3:S37-62. doi: 10.1016/j.cmi.2016.01.007

26. Wang F, Hou HY, Wu SJ, Zhu Q, Huang M, Yin B, et al. Using the Tbag/PHA Ratio in the T-SPOT((R)).TB Assay to Distinguish TB Disease From LTBI in 
an Endemic Area. Int J Tuberc Lung Dis (2016) 20(4):487-93. doi: 10.5588/ ijtld.15.0756

27. Cresswell F, Lange C, van Crevel R. Improving the Diagnosis of Tuberculous Meningitis: Good, But Not Good Enough. Clin Microbiol Infect (2020) 26 (2):134-6. doi: 10.1016/j.cmi.2019.10.009

28. Erdem H, Ozturk-Engin D, Elaldi N, Gulsun S, Sengoz G, Crisan A, et al. The Microbiological Diagnosis of Tuberculous Meningitis: Results of Haydarpasa1 Study. Clin Microbiol Infect (2014) 20(10):O600-8. doi: 10.1111/14690691.12478

29. Thwaites GE, van Toorn R and Schoeman J. Tuberculous Meningitis: More Questions, Still Too Few Answers. Lancet Neurol (2013) 12(10):999-1010. doi: 10.1016/S1474-4422(13)70168-6

30. Kwon JS, Park JH, Kim JY, Cha HH, Kim MJ, Chong YP, et al. Diagnostic Usefulness of Cytokine and Chemokine Levels in the Cerebrospinal Fluid of Patients With Suspected Tuberculous Meningitis. Am J Trop Med Hyg (2019) 101(2):343-9. doi: 10.4269/ajtmh.18-0947

31. Li Z, Du B, Li J, Zhang J, Zheng X, Jia H, et al. Cerebrospinal Fluid Metabolomic Profiling in Tuberculous and Viral Meningitis: Screening Potential Markers for Differential Diagnosis. Clin Chim Acta (2017) 466:3845. doi: 10.1016/j.cca.2017.01.002

32. Pan L, Liu F, Zhang J, Li J, Jia H, Huang M, et al. Genome-Wide MiRNA Analysis Identifies Potential Biomarkers in Distinguishing Tuberculous and Viral Meningitis. Front Cell Infect Microbiol (2019) 9:323. doi: 10.3389/ fcimb.2019.00323

33. Thwaites GE. Advances in the Diagnosis and Treatment of Tuberculous Meningitis. Curr Opin Neurol (2013) 26(3):295-300. doi: 10.1097/WCO. 0b013e3283602814

34. Horne DJ, Kohli M, Zifodya JS, Schiller I, Dendukuri N, Tollefson D, et al. Xpert MTB/RIF and Xpert MTB/RIF Ultra for Pulmonary Tuberculosis and Rifampicin Resistance in Adults. Cochrane Database Syst Rev (2019) 6: CD009593. doi: 10.1002/14651858.CD009593.pub4

35. Kohli M, Schiller I, Dendukuri N, Dheda K, Denkinger CM, Schumacher SG, et al. Xpert((R)) MTB/RIF Assay for Extrapulmonary Tuberculosis and Rifampicin Resistance. Cochrane Database Syst Rev (2018) 8:CD012768. doi: 10.1002/14651858.CD012768.pub2

36. Foppiano Palacios C, Saleeb PG. Challenges in the Diagnosis of Tuberculous Meningitis. J Clin Tuberc Other Mycobact Dis (2020) 20:100164. doi: 10.1016/ j.jctube.2020.100164

37. Wang F, Liu K, Peng J, Luo Y, Tang G, Lin Q, et al. Combination of Xpert MTB/RIF and Tbag/PHA Ratio for Prompt Diagnosis of Active Tuberculosis: A Two-Center Prospective Cohort Study. Front Med (Lausanne) (2020) 7:119. doi: $10.3389 /$ fmed.2020.00119

38. Bosco MJ, Hou H, Mao L, Wu X, Ramroop KD, Lu Y, et al. The Performance of the Tbag/PHA Ratio in the Diagnosis of Active TB Disease in
Immunocompromised Patients. Int J Infect Dis (2017) 59:55-60. doi: 10.1016/j.ijid.2017.03.025

39. Wang F, Hou H, Zhou H, Wu S, Mao L, Huang M, et al. Use of Tbag/PHA Ratio in Distinguishing Tuberculoma From Cancer in Solitary Pulmonary Nodule or Mass. Clin Respir J (2018) 12(3):1174-81. doi: 10.1111/crj.12648

40. Wu X, Huang H, Hou H, Shen G, Yu J, Zhou Y, et al. Diagnostic Performance of a 5-Marker Predictive Model for Differential Diagnosis Between Intestinal Tuberculosis and Crohn's Disease. Inflammation Bowel Dis (2018) 24 (11):2452-60. doi: 10.1093/ibd/izy154

41. Luo Y, Xue Y, Lin Q, Tang G, Yuan X, Mao L, et al. A Combination of Iron Metabolism Indexes and Tuberculosis-Specific Antigen/Phytohemagglutinin Ratio for Distinguishing Active Tuberculosis From Latent Tuberculosis Infection. Int J Infect Dis (2020) 97:190-6. doi: 10.1016/j.ijid.2020.05.109

42. Luo Y, Tan Y, Yu J, Lin Q, Hou H, Mao L, et al. The Performance of Pleural Fluid T-SPOT.TB Assay for Diagnosing Tuberculous Pleurisy in China: A Two-Center Prospective Cohort Study. Front Cell Infect Microbiol (2019) 9:10. doi: $10.3389 /$ fcimb. 2019.00010

43. Luo Y, Tang G, Lin Q, Mao L, Xue Y, Yuan X, et al. Combination of Mean Spot Sizes of ESAT-6 Spot-Forming Cells and Modified Tuberculosis-Specific Antigen/Phytohemagglutinin Ratio of T-SPOT.TB Assay in Distinguishing Between Active Tuberculosis and Latent Tuberculosis Infection. J Infect (2020) 81(1):81-9. doi: 10.1016/j.jinf.2020.04.038

44. Marais S, Thwaites G, Schoeman JF, Torok ME, Misra UK, Prasad K, et al. Tuberculous Meningitis: A Uniform Case Definition for Use in Clinical Research. Lancet Infect Dis (2010) 10(11):803-12. doi: 10.1016/S1473-3099 (10)70138-9

Conflict of Interest: The authors declare that the research was conducted in the absence of any commercial or financial relationships that could be construed as a potential conflict of interest.

Publisher's Note: All claims expressed in this article are solely those of the authors and do not necessarily represent those of their affiliated organizations, or those of the publisher, the editors and the reviewers. Any product that may be evaluated in this article, or claim that may be made by its manufacturer, is not guaranteed or endorsed by the publisher.

Copyright (c) 2021 Luo, Xue, Lin, Mao, Tang, Song, Liu, Wu, Liu, Zhou, Xu, Xiong, Wang, Yuan, Gan, Sun and Wang. This is an open-access article distributed under the terms of the Creative Commons Attribution License (CC BY). The use, distribution or reproduction in other forums is permitted, provided the original author(s) and the copyright owner(s) are credited and that the original publication in this journal is cited, in accordance with accepted academic practice. No use, distribution or reproduction is permitted which does not comply with these terms. 